\title{
Reporte de padres, profesores y cuidadores sobre problemas de comportamiento externalizado de niños y adolescentes en acogimiento residencial versus niños que viven con sus familias
}

\author{
Parent, teacher, and caregiver reports on outsourced behavior \\ problems of children and adolescents in residential care versus \\ children living with their families
}

Martha Patricia FERNÁNDEZ-DAZA ${ }^{1}$ (iD) 0000-0002-8145-0286

\section{Resumen}

Los comportamientos externalizados en niños y adolescentes en acogimiento residencial se presentan con regularidad, convirtiéndose en predictores de conductas delictivas, dinámicas relacionales disfuncionales, consumo de sustancias, entre otros. El objetivo general del presente estudio fue identificar y comparar problemas de comportamiento externalizado de niños y adolescentes en acogimiento residencial y con sus familias, reportados por padres, profesores y cuidadores. La muestra estuvo conformada por 222 adolescentes y preadolescentes entre los 11 y 16 años del Distrito Federal de Caracas y del Estado Miranda-Venezuela. El instrumento utilizado fue el Cuestionario sobre el Comportamiento de Niños y Niñas de 6-18 años. No se encontraron diferencias estadísticamente significativas entre los grupos. Sin embargo, la puntuación en ruptura de normas es más alta en el grupo que vive en acogimiento residencial, contrario a la del grupo control cuya puntuación más alta fue en conducta agresiva. Los hallazgos permiten entrever que, aunque en los reportes de padres, profesores y cuidadores no se encontraron diferencias entre los grupos en relación con el comportamiento externalizado, los puntajes altos pueden concebirse como conductas en riesgo ante las cuales hay que actuar con cautela y hacer seguimiento.

Palabras clave: Conducta infantil; Niño institucionalizado; Niño acogido.

$\checkmark \nabla \nabla$

${ }^{1}$ Universidad Cooperativa de Colombia, Facultad de Psicología, Seccional Santa Marta. Troncal del Caribe. Sector Mamatoco, Santa Marta, Colombia. Correspondence to: M.P. FERNÁNDEZ-DAZA. E-mail: <mafercv@gmail.com>.

Artículo basado en la disertación de M.P. FERNÁNDEZ-DAZA, titulada "Características psicológicas y psicopatológicas de adolescentes venezolanos institucionalizados". Universidad de Granada, 2013.

\section{$\checkmark v$}

How to cite this article

Fernández-Daza, M. P. (2020). Reporte de padres, profesores y cuidadores sobre problemas de comportamiento externalizado de niños y adolescentes en acogimiento residencial versus niños que viven con sus familias. Estudos de Psicologia (Campinas), 37 , e190019. http://dx.doi.org/10.1590/1982-0275202037e190019 


\begin{abstract}
Externalized behaviors in children and adolescents in residential care are regularly presented and taken as predictors of criminal behavior, dysfunctional relational dynamics, substance use, among others. The general objective of this study was to identify and compare problems of externalized behavior of children and adolescents in residential care and living with their families as reported by parents, teachers, and caregivers. The sample consisted of 222 adolescents and preadolescents between 11 and 16 of the Caracas Federal District and Miranda State, in Venezuela. The instrument used was the Child Behavior Check-List. No statistically-significant differences were found between the groups. However, the breaking score is higher for the group that lives in residential care; contrary to the control group, whose highest score was in aggressive behavior. The findings allow to see that, although in the reports of parents, teachers, and caregivers no differences were found between the groups in relation to their externalized behavior, the high scores can be conceived as risky behaviors before which one must act with caution and follow up.
\end{abstract}

Keywords: Child behavior, Child, Institutionalized; Child, Foster.

A nivel mundial, los comportamientos externalizados de niños y adolescentes tienen alta prevalencia; así mismo, las consecuencias que generan revelan la importancia de su detección en edades tempranas. Se conciben como aquellos problemas de conducta que se dirigen al exterior, vinculan a otras personas y ocasionan daño. Los comportamientos externalizados se clasifican en comportamiento agresivo y comportamiento de ruptura de normas. Además, están vinculados a comportamientos desadaptativos tales como: falta de obediencia, conductas destructivas, agitación psicomotora, agresiones físicas, delincuencia, entre otros. Lo que a su vez repercute en la dinámica familiar, el desempeño académico y en la interacción social con hermanos, compañeros, docentes (Delgado, 2013; Hewitt, 2016).

Los comportamientos externalizados han sido ampliamente documentados en la literatura (Hewitt, 2016; Hewitt-Ramírez \& Moreno-Méndez, 2018; Rescorla, Althoff, Ivanova, \& Achenbach, 2019; Rescorla et al., 2012). También se ha indicado que presentan comorbilidad con otras patologías como ansiedad y depresión, asimismo con otros problemas psiquiátricos, psicológicos, psicopatológicos, del desarrollo, déficits neuropsicológicos, exclusión social y estigmatización (Delgado, 2013; Fernández-Daza, 2013; FernándezDaza, 2012a; Fernández-Daza, 2012b; Hewitt, 2016; Sainero, Valle, \& Bravo, 2015). Y se ha señalado que el estrés parental y el nivel socioeconómico tienen relación con la presencia de comportamientos externalizados (Ulloa, Cova, \& Bustos, 2017).

Por otro lado, el acogimiento residencial es una forma de cuidado alternativo que se utiliza para proteger a la población infantil sin cuidado parental. En este caso, la responsabilidad recae sobre el estado o asociaciones civiles y entidades de protección. El cuidado se otorga dentro de un contexto institucional o residencial, donde los niños y adolescentes permanecen bajo la supervisión de cuidadores que no forman parte de su familia biológica.

Desde hace mucho que se ha reflexionado sobre las consecuencias del acogimiento residencial, en este sentido se recuerdan los trabajos de Spitz, Goldfarb, Bowlby y Ainsworth citados por Fernández-Daza (2013). El primero se motivó por analizar la relación entre los vínculos afectivos tempranos y sus carencias, producto del acogimiento residencial; el segundo por los efectos de la privación psicosocial; el tercero y la cuarta sobre la privación maternal, para finalmente Bowlby hacer énfasis con su teoría del apego.

Pero, afortunadamente, estas prácticas inadecuadas del sistema de protección infantil generalmente se acompañan de medidas alternativas más viables y de propuestas específicas de tratamiento (Monzó-Fenollar \& Ballespí-Solá, 2013; Fernández-Daza, 2012a; Fernández-Daza, 2012b; Fernández-Daza, 2013).

Se ha manifestado que las condiciones familiares adversas, el abuso y la negligencia durante la infancia representan importantes factores de riesgo para la aparición de problemas emocionales y de comportamiento (González-García et al., 2017). 
A pesar de que las directrices sobre el acogimiento residencial hacen énfasis en que los bajos recursos económicos no son motivo para el ingreso de los niños y adolescentes a esta modalidad de cuidado, estudios recientes reportan que la realidad es otra, principalmente, en los países latinoamericanos (Fernández-Daza, 2013).

Los problemas de comportamiento externalizado en niños que se encuentran en acogimiento residencial han sido asociados a diversos factores, entre ellos: la edad de ingreso a la institución, las características tanto de las instituciones como de los cuidadores, la duración del acogimiento, la comunicación e interacción con los progenitores y las experiencias traumáticas como el maltrato, el abuso y el desamparo (Delgado, 2013; Fernández-Daza, 2013; Fernández-Fernández, 2014; Gearing, Schwalbe, MacKenzie, Brewer, \& Ibrahim, 2015; Moreno, Hernández, \& Suárez, 2016).

A su vez, los problemas de comportamiento externalizado en niños y adolescentes en acogimiento residencial se han relacionado con múltiples riesgos y necesidades psicosociales (Leloux-Opmeer, Kuiper, Swaab, \& Scholte, 2017) adicciones y conductas delictivas (González, 2018); problemáticas de adaptación y socialización (Deambrosio, Vázquez, Arán-Filippetti, \& Román, 2018; Llanos, 2013); problemas de conducta, emocionales y problemas internalizantes (Cantón-Cortés \& Cortés, 2015; Juárez, 2016; Leloux-Opmeer, Kuiper, Swaab, \& Scholte, 2016; Sánchez, 2015) y con problemas de apego (Muzi \& Bizzi, 2018).

Estudios recientes plantean que para los niños que residen en entornos institucionales, el personal actúa como cuidador principal y a menudo proporciona evaluaciones de la psicopatología infantil. Pero, existe poca investigación sobre cómo y cuándo los cuidadores están más preparados para informar sobre la salud mental de los jóvenes, lo que sugiere la necesidad de investigación adicional en esta área crítica (Gearing et al., 2015).

En adición a ello, es incipiente la investigación en Latinoamérica sobre niños y adolescentes en acogimiento residencial; algunos estudios recientes son: Ariza-Barrios, Novoa-Rusinque y González-Puentes (2016). Sin embargo, ninguno considera los problemas de comportamiento externalizado como su objeto de estudio.

En este contexto, resulta relevante conocer los problemas comportamentales de tipo externalizados de los niños y adolescentes venezolanos que se encuentran en acogimiento residencial.

\section{Método}

\section{Participantes}

La muestra fue de 222 adolescentes y preadolescentes entre los 11 y 16 años del Distrito Federal Caracas y del Estado Miranda-Venezuela, los cuales estudiaban en colegios públicos, privados o subsidiados, la media fue de 13.3 años $(D T=1.24) ; 124$ de los participantes eran mujeres y 98 varones. Los grupos se conformaron así: el grupo integrado por niños y adolescentes en acogimiento residencial $(N=111)$ y el grupo Control $(N=111)$. Cada grupo tenía 62 mujeres y 49 hombres (edad: $M=13.46 ; D T=1.41$; edad: $M=$ 13.14; $D T=1.03$, respectivamente). Aunque se ha señalado la importancia de la detección de los problemas de comportamiento externalizado en edades tempranas, el rango de edad escogida para el estudio se realizó por conveniencia, debido a que la mayoría de los niños y adolescentes en acogimiento residencial se encontraban en estas edades.

Los criterios de inclusión o exclusión fueron: estar o no en acogimiento residencial en centros de protección bajo la tutela del Estado o asociaciones civiles, tener entre 11 y 16 años, y estar escolarizados entre los cursos quinto de primaria y noveno de bachillerato, ambos inclusive.

En lo que respecta a las "características de la muestra en acogimiento residencial", se observó que los motivos de ingreso al programa de acogimiento eran: orfandad, situación de calle, abandono o retiro de 
la custodia a los padres. La mayoría tenía más de un año viviendo en la institución y había ingresado entre los 8 y 11 años.

Sobre las "características de las instituciones": 2 eran asociaciones civiles sin fines de lucro y 3 entidades de protección del Estado. La totalidad de las instituciones acogían a niños y adolescentes cuyos derechos habían sido vulnerados o amenazados. 3 de las instituciones eran de naturaleza religiosa y 2 no tenían adscripción confesional. Las 5 instituciones acogían niños y adolescentes de 0 a18 años.

En cuanto a las "características de la muestra del grupo normalizado", estaba conformado por preadolescentes y adolescentes que pertenecían a 4 colegios de educación normalizada (3 subsidiados y 1 privado), dos de los cuales eran religiosos. La muestra de participantes en acogimiento residencial se conformó con aquellos cuya valoración estuvo completa. Para integrar el grupo control, los participantes se igualaron en número, edad y sexo con el grupo en acogimiento residencial.

\section{Instrumento}

El Achenbach System of Empirically Based Assessment permite la valoración de manera efectiva del comportamiento adaptativo y no adaptativo de niños y adolescentes. Dentro de este sistema se encuentra el Cuestionario sobre el Comportamiento de Niños y Niñas de 6-18 años (Child Behavior Check-List [CBCL]); (Achenbach \& Rescorla, 2001). El CBCL se ha diseñado para recibir información de diferentes informantes (padres, profesores y cuidadores) sobre el comportamiento de niños y adolescentes. El instrumento consta de dos partes, la segunda parte, que es la que interesa para este estudio, está conformada por 113 ítems y permite obtener un perfil de comportamiento del niño, a través de 8 escalas de "banda estrecha": aislamiento, ansiedad/depresión, síntomas somáticos, problemas sociales, problemas de atención, problemas de pensamiento, conducta de ruptura de normas y conducta agresiva. Hay otras dos escalas de "banda ancha", una de ellas es la de síndromes externalizados (conducta de ruptura de normas y conducta agresiva).

En cuanto a la confiabilidad del $C B C L$, se ha reportado de 0.79 para las escalas relacionadas con las competencias sociales; de 0.78 a 0.97 para cada una de las escalas de síndromes o comportamientos específicos y de 0.95 para las escalas totales (Hewitt-Ramírez \& Moreno-Méndez, 2018).

Para el presente estudio se utilizó el $C B C L$ en sus formas padres y profesores. Con el objetivo de validar el contenido, se utilizó el juicio de 4 expertos venezolanos en el campo de la Psicología para que determinaran la adecuada comprensión de los ítems del CBCL. Se tuvieron en cuenta los siguientes criterios: claridad, pertinencia, lenguaje, precisión y metodología; luego se hizo el análisis y se observó que obtuvo una puntuación alta en contenido y forma.

\section{Procedimiento}

Se realizó la validez de contenido, luego la búsqueda y selección de los colegios y las instituciones de protección de niños y adolescentes en acogimiento residencial. Se contactó con cada una y se les informó sobre el estudio a realizar. Al obtener el consentimiento de las instituciones, se seleccionó la muestra y se envió el consentimiento informado a los padres y profesores de los niños que vivían con sus familias. En todos los casos se trabajó con los niños y adolescentes que cumplieran con los criterios de inclusión. Finalmente, se entregó el CBCL para que cada niño lo llevara a casa y escuela para ser respondido por padres y profesores. En el caso de los niños en acogimiento residencial, se entregó a los cuidadores y profesores.

El análisis de resultados se realizó con el paquete estadístico IBM ${ }^{\circledR}$ SPSS$^{\circledR}$ (versión 18), se extrajo Chicuadrado para las variables cualitativas y se realizó un análisis de la varianza multivariado de tres factores 
para la comparación entre grupos, atendiendo al grupo de pertenencia (acogimiento residencial y grupo que vive con familia biológica), sexo y nivel escolar. A través del modelo factorial se analizaron los efectos de los factores inter-sujetos fijos en las variables dependientes, así como sus respectivas interacciones. Los factores fijos eran: el grupo, sexo y el nivel escolar y las variables dependientes estuvieron constituidas por el desempeño en el instrumento aplicado.

Vale indicar que los resultados presentados contemplan el reporte de diferentes informantes (padres, profesores y cuidadores), teniendo en cuenta que gran parte de la literatura existente expone la importancia de observar el comportamiento de los niños y adolescentes en el propio entorno donde se desenvuelven e interactúan debido a que sus patrones comportamentales podrían ser diferentes dependiendo del ambiente, las personas que le rodean, entre otros. Además, la evaluación multi-informante refleja las diferentes percepciones de los informantes por lo que proporciona un conocimiento más amplio de su comportamiento, facilitando un diagnóstico más certero y su intervención (Achenbach, McConanghy, \& Howell, 1987; Achenbach, Ivanova, \& Rescorla, 2017; Ivanova et al., 2018; Maganto, 2010; Poch et al., 2008; Steinberg \& Morris, 2001).

\section{Resultados}

Tal como se muestra en la Tabla 1, en el reporte de padres, profesores y cuidadores sobre el comportamiento de los jóvenes $(\mathrm{CBCL})$, no hubo diferencias estadísticamente significativas entre los grupos. Vale apreciar que la puntuación en ruptura de normas es más alta en el grupo que vive en acogimiento residencial, contrario a las del grupo control cuya puntuación más alta fue en conducta agresiva.

En segundo lugar, se hace alusión al análisis multivariado del factor Sexo. Se puede comprobar que hubo diferencias estadísticamente significativas en todas las escalas evaluadas en los informes de padres, profesores y cuidadores. Específicamente, las puntuaciones más elevadas correspondieron al sexo masculino. (Tabla 2).

En tercer lugar, se realizó el análisis multivariado del factor curso escolar, cuyos resultados se recogen en la Tabla 3. En esta oportunidad, no hubo diferencias estadísticamente significativas. En todos los casos, los participantes de primaria puntuaron más bajo que los jóvenes de bachillerato.

En cuarto lugar, se presentan los resultados del análisis de varianza multivariado realizado entre las variables de estudio y los factores Grupo x Sexo. Los resultados mostraron que no hubo efectos de interacción estadísticamente significativos. La Tabla 4 permite una inspección detallada de estos resultados.

En quinto lugar, en lo que respecta al análisis de varianza multivariado realizado entre las variables de estudio y los factores Grupo por Curso, que se presenta en la Tabla 5, no hubo efectos de interacción, pero, las puntuaciones más altas eran las de los participantes de Primaria del grupo en acogimiento residencial y los participantes de Bachillerato del grupo control.

Tabla 1

Problemas de comportamiento externalizado (Comparación entre grupos)

\begin{tabular}{|c|c|c|c|c|c|c|}
\hline \multirow{3}{*}{ Escalas } & \multicolumn{4}{|c|}{ Grupos } & \multirow[b]{3}{*}{$F$} & \multirow[b]{3}{*}{$p$} \\
\hline & \multicolumn{2}{|c|}{ Acog Residenc $(N=111)$} & \multicolumn{2}{|c|}{ Control $(N=111)$} & & \\
\hline & M & $D T$ & M & $D T$ & & \\
\hline CBCL - Ruptura de normas & 4,01 & 4,38 & 3,88 & 4,07 & 0,787 & 0,376 \\
\hline CBCL - Conducta agresiva & 7,05 & 7,83 & 7,26 & 5,87 & 0,539 & 0,464 \\
\hline CBCL - Síndromes Externalizados & 11,06 & 11,81 & 11,14 & 9,54 & 0,679 & 0,411 \\
\hline
\end{tabular}

Nota: DT: Desviación Típica; M: Média. 
Tabla 2

Análisis multivariado (Sexo y Problemas de Comportamiento Externalizado)

\begin{tabular}{|c|c|c|c|c|c|c|}
\hline \multirow{3}{*}{ Escalas } & \multicolumn{4}{|c|}{ Sexo } & \multirow[b]{3}{*}{$F$} & \multirow[b]{3}{*}{$p$} \\
\hline & \multicolumn{2}{|c|}{ Femenino $(N=124)$} & \multicolumn{2}{|c|}{ Masculino $(N=98)$} & & \\
\hline & M & $D T$ & $M$ & $D T$ & & \\
\hline CBCL - Ruptura de normas & 3,20 & 3,07 & 4,87 & 5,14 & 6,243 & $0,013^{*}$ \\
\hline CBCL - Conducta agresiva & 6,15 & 5,83 & 8,42 & 7,85 & 5,229 & $0,023 *$ \\
\hline CBCL - Síndromes Externalizados & 9,36 & 8,50 & 13,29 & 12,57 & 6,059 & $0,015^{*}$ \\
\hline
\end{tabular}

Nota: *Diferencias significativas entre grupos. DT: Desviación Típica; M: Média.

Tabla 3

Análisis multivariado (Nivel Escolar y Problemas de Comportamiento Externalizado)

\begin{tabular}{|c|c|c|c|c|c|c|}
\hline \multirow{3}{*}{ Escalas } & \multicolumn{4}{|c|}{ Nivel Escolar } & \multirow[b]{3}{*}{$F$} & \multirow[b]{3}{*}{$p$} \\
\hline & \multicolumn{2}{|c|}{ Primaria $(N=56)$} & \multicolumn{2}{|c|}{ Bachillerato $(N=166)$} & & \\
\hline & M & $D T$ & M & $D T$ & & \\
\hline CBCL - Ruptura de normas & 3,51 & 4,13 & 4,08 & 4,21 & 0,486 & 0,487 \\
\hline CBCL - Conducta agresiva & 6,85 & 7,45 & 7,25 & 6,68 & 0,047 & 0,828 \\
\hline CBCL - Síndromes Externalizados & 10,37 & 11,37 & 11,345 & 10,42 & 0,173 & 0,678 \\
\hline
\end{tabular}

Nota: DT: Desviación Típica; M: Média.

Tabla 4

Interacción Grupo x Sexo respecto a Problemas de Comportamiento Externalizado

\begin{tabular}{|c|c|c|c|c|c|c|c|c|c|c|}
\hline \multirow{4}{*}{ Escalas } & \multicolumn{9}{|c|}{ Grupos } & \multirow[b]{4}{*}{$p$} \\
\hline & \multicolumn{4}{|c|}{ Acogimiento Residencial } & \multicolumn{4}{|c|}{ Control } & \multirow[b]{3}{*}{$F$} & \\
\hline & \multicolumn{2}{|c|}{ Femenino } & \multicolumn{2}{|c|}{ Masculino } & \multicolumn{2}{|c|}{ Femenino } & \multicolumn{2}{|c|}{ Masculino } & & \\
\hline & $M$ & $D T$ & M & $D T$ & M & $D T$ & M & $D T$ & & \\
\hline CBCL - Ruptura de normas & 3,13 & 3,14 & 5,12 & 5,40 & 3,28 & 3,06 & 4,66 & 5,02 & 0,062 & 0,804 \\
\hline CBCL - Conducta agresiva & 5,45 & 5,85 & 9,08 & 8,70 & 6,85 & 4,98 & 7,79 & 6,13 & 0,002 & 0,961 \\
\hline CBCL - Síndromes Externalizados & 8,58 & 8,99 & 14,20 & 14,10 & 10,13 & 8,04 & 12,45 & 11,15 & 0,457 & 0,500 \\
\hline
\end{tabular}

Note: DT: Desviación Típica; M: Média.

Tabla 5

Interacción Grupo x Nivel Educativo respecto a Problemas de Comportamiento Externalizado.

\begin{tabular}{|c|c|c|c|c|c|c|c|c|c|c|}
\hline \multirow{4}{*}{ Escalas } & \multicolumn{8}{|c|}{ Grupos } & \multirow[b]{4}{*}{$F$} & \multirow[b]{4}{*}{$p$} \\
\hline & \multicolumn{4}{|c|}{ Acogimiento Residencial } & \multicolumn{4}{|c|}{ Control } & & \\
\hline & \multicolumn{2}{|c|}{ Primaria } & \multicolumn{2}{|c|}{ Bachillerato } & \multicolumn{2}{|c|}{ Primaria } & \multicolumn{2}{|c|}{ Bachillerato } & & \\
\hline & M & $D T$ & M & $D T$ & M & $D T$ & M & $D T$ & & \\
\hline CBCL - Ruptura de normas & 3,95 & 4,61 & 4,04 & 4,29 & 2,61 & 2,77 & 4,13 & 4,25 & 1,439 & 0,232 \\
\hline CBCL - Conducta agresiva & 7,66 & 8,52 & 6,74 & 7,49 & 5,17 & 4,18 & 7,68 & 6,09 & 2,850 & 0,093 \\
\hline CBCL - Síndromes Externalizados & 11,61 & 12,93 & 10,78 & 11,26 & 7,78 & 6,66 & 11,81 & 9,91 & 2,442 & 0,120 \\
\hline
\end{tabular}

Note: DT: Desviación Típica; M: Média.

\section{Discusión}

Los problemas de comportamiento externalizado en niños y adolescentes que viven en acogimiento residencial han sido motivo de interés recientemente, más en países anglosajones y europeos que en Latinoamérica. El objetivo principal del presente estudio es el reporte de problemas de comportamiento 
externalizado en niños y adolescentes que viven en acogimiento residencial y con sus familias biológicas, informado por padres, profesores y cuidadores.

Los resultados encontrados en la presente investigación permiten indicar que entre los grupos participantes no hubo diferencias en los síndromes externalizados. Vale indicar que, contrario a lo reportado, fue el grupo de niños y adolescentes que convive con sus familias el que tiene una tendencia a la alta presentación de este tipo de comportamientos. A la luz de los presentes resultados, hay algunos aspectos que se podrían considerar: 1) qué tan capacitados estaban los cuidadores para informar sobre el comportamiento externalizado de los niños y adolescentes en acogimiento residencial, en el sentido de diferenciar un comportamiento adecuado para su desarrollo evolutivo de un comportamiento patológico; 2) Qué tanto conocía el cuidador al niño; 3) Variables como el tiempo que tiene el cuidador en la institución, frecuencia de cuidado (todos los días o algunos días a la semana), si el cuidado ha sido estable o esporádico o la percepción que tiene del niño pudieran haber influido en el reporte del cuidador; 4) Tipo y grado de vínculo entre el niño y su cuidador; 5) Factores protectores en los niños que redujeron la probabilidad de ocurrencia de problemáticas de comportamiento externalizado.

Los presentes hallazgos están en la línea con lo constatado en estudios previos que señalan que gran parte de la población en acogimiento residencial estuvieron dentro del rango de la normalidad en cuanto a los comportamientos externalizados (46,4\%) (Fernández-Molina, Del Valle, Fuentes, Bernedo, \& Bravo, 2011). También vale precisar que, aunque la actual investigación se hizo con el reporte de padres, cuidadores y profesores acerca del comportamiento externalizado de niños y adolescentes que viven en acogimiento residencial y con sus familias biológicas, coincide con hallazgos donde los informes de la población en acogimiento residencial de los problemas de comportamiento externalizado tampoco mostraron significación estadística (Sainero, Del Valle, \& Bravo, 2015).

Independientemente de que no se hayan encontrado diferencias significativas en los comportamientos externalizados entre los niños y adolescentes en cuidado residencial y aquellos que conviven con sus familias, es preciso llamar la atención sobre la importancia de la observación y el seguimiento del comportamiento de este tipo, porque existe un gran cuerpo de evidencia que reporta hallazgos en este sentido. Por ejemplo, se ha encontrado que un 33,9\% de los adolescentes en acogimiento residencial se encontraban en el rango clínico para síndromes externalizados, específicamente el 23,2\% para conducta delincuente y el 17,9\% para conducta agresiva (Fernández-Molina et al., 2011). Igualmente, Swinton (2011) concluyó que niños que ya habían sido adoptados mostraron proporciones más altas en las puntuaciones de comportamientos de tipo externalizado que los que vivían con sus familiares. Sainero et al. (2015) encontraron que el $65 \%$ de los niños en acogimiento residencial tenía diagnóstico para trastornos de tipo externalizado.

Los hallazgos guardan ciertas diferencias con estudios recientes de Delgado (2013) que indican que en los reportes de padres, profesores y cuidadores los participantes que viven en las instituciones presentan más problemas de comportamiento que los preadolescentes y adolescentes que conviven con sus familias. Se presume que estas diferencias pudieran ser porque en el presente estudio solo se profundizó en el reporte del comportamiento de tipo externalizado, mientras que Delgado (2013) analizó también el reporte de otros tipos de comportamientos como los problemas internalizados. Asimismo, en los dos estudios realizados por la autora, las diferencias culturales pudieran ser objeto de las discrepancias encontradas, acompañado de las edades, el nivel escolar y el tamaño de la muestra; por ejemplo, en el primer estudio la muestra de niños y adolescentes en acogimiento residencial fue de 26 contra 111 en el presente estudio y la muestra de niños que convivían con sus padres fue de 45 contra 111 en el estudio actual. $Y$ en su segundo estudio fueron (41 contra 111) y (100 contra 111).

Y en un estudio que analizó tres metaanálisis sobre los comportamientos externalizados e internalizados y la percepción del cuidado de niños en acogimiento residencial versus niños en acogimiento familiar, 
concluyó que los niños en cuidado familiar tuvieron siempre mejores experiencias y menos problemas en las tres variables en comparación con los niños en cuidado residencial (Li, Chng, \& Chu, 2017).

En consecuencia, es preciso estar atentos al acogimiento residencial puesto que la mayoría de los niños que ingresan tienen necesidades afectivas y psicosociales serias. En este sentido, vale indicar que, para la detección de riesgos y necesidades del acogimiento, los profesionales a cargo deben tener información suficiente del niño y su familia, el historial completo del acogimiento, conocer sobre el desarrollo evolutivo sano y la influencia de las características personales y socioculturales. No obstante, son muchas las instituciones que no llevan un registro cuidadoso de la población que ingresa.

Por otro lado, en edades tempranas los pares y cuidadores tempranos pueden constituirse en modelos vinculares que ejercen un papel mediador sobre la conducta del niño o adolescente. Es por ello por lo que, ante problemas de comportamiento externalizado, las acciones preventivas y correctivas deben hacerse lo más pronto posible, más en contextos de alto riesgo como los residenciales, donde el número de niños generalmente es alto. Así, se concuerda con algunos estudios que alertan sobre las diversas representaciones de apego y la utilidad potencial de monitorear su calidad, porque puede provocar o perpetuar conductas agresivas o delictivas entre los adolescentes, en lugar de promover el ajuste social (Delgado, 2013; Muzi \& Bizzi, 2018).

Al detallar la subescala ruptura de normas en los presentes resultados se puede apreciar que la puntuación es más alta en el grupo que vive en acogimiento residencial, contrario a las del grupo control cuya puntuación más alta correspondió a conducta agresiva. Tal como han evidenciado otras investigaciones que también indican que el porcentaje más alto de adolescentes en acogimiento residencial se encuentra en la subescala de conducta delincuente (ruptura de normas) (23,2\%) (Fernández-Molina et al., 2011). Esto también va en consonancia con estudios realizados con menores en sistemas de protección que han sido víctimas de maltrato (Fernández-Fernández, 2014). Razones por las cuales se coincide con Leloux-Opmeer et al. (2016) quienes exponen que los entornos basados en la familia se consideran el tipo de cuidado preferido cuando se requiere una colocación fuera del hogar.

Es importante también que las instituciones de acogimiento cuenten con un equipo de salud y atención psicosocial que les apoye en el cuidado de los niños, además como una medida de prevención de riesgos y promoción de salud infantil. Sobre el particular, se ha afirmado que los niños en acogimiento residencial tienen una necesidad especial de atención especializada en enfermedades mentales, problemas de comportamiento y escolares / de aprendizaje (González, 2018; Leloux-Opmeer et al., 2017). Investigaciones reportan que, de 1216 niños, menos del 50\% estaban recibiendo algún tipo de tratamiento de salud mental, a pesar de que el $61 \%$ se identificó dentro del rango clínico en algunas de las escalas de banda ancha del CBCL (GonzálezGarcía et al., 2017).

Adicionalmente, sería interesante conocer si existen discrepancias o semejanzas entre los reportes de los padres con los reportes de profesores y cuidadores que pudieran estar influyendo en las puntuaciones reflejadas, ya que la calidad de la relación pudiera asociarse con el reporte del comportamiento. O la duración del acogimiento residencial, porque como se ha dicho, a mayor tiempo en la institución los problemas externalizados se agudizan; en consecuencia, el reporte de los cuidadores sobre el comportamiento de los niños y adolescentes se agrava, coincidiendo con Delgado (2013).

El enfoque actual de la discusión es sobre la mayor vulnerabilidad de los niños y adolescentes en acogimiento residencial en comparación con los que viven con su familia; sin embargo, los resultados encontrados en el presente estudio no corroboran esta hipótesis. Algunos factores que pudieran haber influido en los resultados pueden estar asociados a: (a) las características propias de las instituciones (número de niños, número de cuidadores, equipo de trabajo, tamaño de la institución, número de niños x cuidador); (b) las características propias de los niños (edad actual, edad de ingreso, sexo, duración en la institución, razones de 
ingreso, experiencia previa, número de acogidas, experiencias de acogida, adaptación al sistema de acogida, factores de riesgo y protección, redes de apoyo, servicios de atención a su salud física y mental, entre otras) y (c) las características propias del informante (vinculación afectiva con el niño, calidad del cuidado, tiempo que conoce al niño, disponibilidad, etc.).

Sobre otro particular, valdría la pena analizar los problemas de comportamiento externalizado en relación con la edad. Es probable que estos se agudicen a medida que la edad avanza, tal como reportan ciertos estudios (González, 2018; Sainero, Del Valle, López, \& Bravo, 2013).

Por otra parte, aunque el CBCL ha mostrado ser un instrumento útil para la evaluación del comportamiento externalizado de niños en acogimiento residencial, esta debe complementarse con otras formas de valoración más específicas de tal forma que permitan un conocimiento más amplio y certero del comportamiento; en este sentido, se concuerda con Gearing et al., (2015).

En cuanto al sexo, del total de la muestra hubo diferencias estadísticamente significativas en todas las escalas del CBCL en los reportes de padres, profesores y cuidadores. Específicamente, el sexo masculino puntuó más alto en ruptura de normas, conducta agresiva y síndromes externalizados. Los presentes hallazgos son similares a investigaciones que han evidenciado que los varones pueden estar en un mayor riesgo de problemas de comportamiento (Ernst, 2000), ya que pueden ser más agresivos que las mujeres, lo que hace que los problemas de conducta sean más probables. También son concordantes con estudios recientes que han encontrado que los problemas externalizados se exhiben con mayor frecuencia en los niños que en las niñas (Hewitt, 2016; Hewitt-Ramírez \& Moreno-Méndez, 2018; Rescorla, Althoff, Ivanova, \& Achenbach, 2019; Rescorla et al., 2012). Igualmente, con los resultados de estudios con niños en acogimiento residencial que plantean que las niñas tienden a sufrir más de problemas internalizantes (Jimeno, 2016).

Lo que muestra alguna discordancia con estudios que indican que el sexo no fue un factor predictivo para los problemas de comportamiento (Pérez, 2016). Pero son coincidentes con otros cuando afirman que los adoptados masculinos que previamente vivieron en acogimiento residencial y actualmente vivían con sus familias mostraron un comportamiento externalizante significativamente mayor que las mujeres adoptadas (Swinton, 2011). Los hallazgos del presente estudio pudieran ser porque las niñas que conformaron la muestra tienen un temperamento más suave, son más tranquilas y menos impulsivas que los niños. En este sentido, valdría la pena revisar para próximas oportunidades si fue el grupo de varones en acogimiento residencial el que presentó más problemas de comportamiento externalizado o aquellos que vivían con sus padres.

En la investigación también se encontró que, al comparar los grupos en cuanto al nivel escolar, no hubo diferencias estadísticamente significativas respecto a los problemas de comportamiento evaluados mediante el CBCL. Sin embargo, sí se aprecia que las puntuaciones más altas las muestra el grupo de bachillerato. En este sentido, sería recomendable hacer seguimiento de la evolución de su comportamiento porque estos puntajes elevados son un indicativo de que la población está en riesgo de presentar en el futuro problemas de comportamiento externalizado.

Los hallazgos en el presente estudio deben ser interpretados con cautela por: (a) el carácter transversal de la investigación, por lo que sus resultados no son generalizables; (b) la dificultad en el acceso a la población en acogimiento residencial; (c) la escases de instrumentos validados en la población venezolana; (d) la dificultad para el acceso a la base de datos personal de los niños y adolescentes en acogimiento residencial con información que permitiera complementar los resultados, incluso la ausencia de registros tales como edad de ingreso a la institución, condiciones físicas y de salud mental, duración del acogimiento residencial, vinculación con la familia, calidad del contacto, entre otros. También sería conveniente analizar las características propias de los cuidadores tales como edad, sexo, años de experiencia, tiempo en la institución, porque estas pueden influir en la calidad de la atención de los niños en acogimiento y, por ende, en el reporte del comportamiento externalizado. 
Se resalta la escasez de estudios de niños en acogimiento residencial en América Latina y se desconocen estudios publicados de niños en acogimiento residencial en Venezuela, lo cual fue una limitación al momento de contar con referentes que permitieran puntos de comparación, pero también es una de las mayores fortalezas porque podría enriquecer el trabajo investigativo y orientaría futuras líneas de acción en Latinoamérica.

Se considera que un aporte importante del presente estudio es proporcionar información realmente útil relacionada con el comportamiento de los niños y adolescentes que viven en acogimiento residencial, principalmente al compararlo con aquellos que conviven con sus padres. Adicionalmente, es relevante para el diseño y ejecución de propuestas y programas de intervención dirigidos a niños y adolescentes en acogimiento residencial que les permitan su atención integral en vista de que se trata de una de las poblaciones más vulnerables.

\section{Referencias}

Achenbach, T. M., Ivanova, M. Y., \& Rescorla, L. A. (2017). Empirically based assessment and taxonomy of psychopathology for ages 11/2-90+ years: developmental, multi informant, and multicultural findings. Comprehensive Psychiatry, 79, 4-18.

Achenbach, T. M., McConanghy, S. H., \& Howell, C. T. (1987). Child/adolescent behavioral and emotional problems: implications of cross-informant correlations for situational specificity. Psychological Bulletin, 101, 213-232.

Achenbach, T. M., \& Rescorla, L. A. (2001). Manual for ASEBA School-Age Forms \& Profiles. Burlington: University of Vermont.

Ariza-Barrios, J. M., Novoa-Rusinque, P. A., \& González-Puentes, D. C. (2016). Condiciones de vulnerabilidad social y problemas internalizantes en niños institucionalizados (Disertación inédito). Universidad de la Sabana.

Cantón-Cortés, D., \& Cortés, M. R. (2015). Child sexual abuse consequences: a review of intervening variables. Anales de Psicología, 31(2), 552-561.

Deambrosio, M., Vázquez, M., Arán-Filippetti, V., \& Román, F. (2018). Efectos del Maltrato en la Neurocognición: un estudio en niños maltratados Institucionalizados y no Institucionalizados. Revista Latinoamericana de Ciencias Sociales, Niñez y Juventud, 16(1), 239-253.

Delgado, L. (2013). Infancia y adolescencia en acogimiento residencial (Tesis de doctorado inédito). Universidad Autónoma de Barcelona.

Ernst, J. L. (2000). Genetic and environmental influences of maternal psychosocial and antisocial tendencies on the development, stability, and continuity of problema behaviors in adoptees from the Texas Adoption Project: a life course investigation of risk, resilience, and vulnerability (Unpublished master's doctoral). University of Texas.

Fernández-Daza, M. P. (2012a). Funcionamiento ejecutivo en población infantil institucionalizada. V Congreso Internacional y x Nacional de Psicología Clínica. Santander.

Fernández-Daza, M. P. (2012b). Institucionalización infantil en sistemas de protección. Visión retrospectiva de un problema vigente. V Congreso Internacional y x Nacional de Psicología Clínica. Santander.

Fernández-Daza, M. P. (2013). Características psicológicas y psicopatológicas de adolescentes venezolanos institucionalizados (Tesis de doctorado inédito). Universidad de Granada.

Fernández-Fernández, M. (2014). Maltrato infantil: un estudio empírico sobre variables psicopatológicas en menores tutelados (Tesis doctoral inédito). Universidad de Murcia.

Fernández-Molina, M., Del Valle, J. F., Fuentes, M. J., Bernedo, I. M., \& Bravo, A. (2011). Problemas de conducta de los adolescentes en acogimiento preadoptivo, residencial y con familia extensa. Psicothema, 23, 1-6.

Gearing, R. E., Schwalbe, C. S., MacKenzie, M. J., Brewer, K. B., \& Ibrahim, R. W. (2015). Assessment of adolescent mental health and behavioral problems in institutional care: discrepancies between staff-reported $C B C L$ scores and adolescent-reported YSR scores. Administration and Policy in Mental Health and Mental Health Services Research, 42(3), 279-287.

González, P. (2018). El acogimiento residencial infantil en Tenerife: perfiles atendidos, programas de intervención y evaluación de resultados (Tesis de doctorado inédito). Universidad de Tenerife.

González-García, C., Bravo, A., Arruabarrena, I., Martín, E., Santos, I., \& Del Valle, J. F. (2017). Emotional and behavioral problems of children in residential care: screening detection and referrals to mental health services. Children and Youth Services Review, 73, 100-106.

Hewitt, N. (2016). Predicción de los problemas de banda ancha y banda estrecha mediante la integración de los modelos transaccional, cognitivo-social y de regulación emocional (Tesis de doctorado inédito). Universidad de Granada. 
Hewitt-Ramírez, N., \& Moreno-Méndez, J. (2018). Intervención psicológica para comportamientos externalizados e internalizados en niños de 8 a 12 años. Revista de Psicología Clínica con Niños y Adolescentes, 5(2), 37-42.

Ivanova, M. Y., Achenbach, T. M., Rescorla, L. A., Guo, J., Althoff, R. R., Kan, K. J., ... Rocha, M. M. (2018). Testing syndromes of psychopathology in parent and youth ratings across societies. Journal of Clinical Child \& Adolescent Psychology, 24, 1-14.

Jimeno, M. V. (2016). Experiencias traumáticas en la infancia y su influencia sobre el desarrollo afectivo-social y la memoria autobiográfica en adolescentes institucionalizados comparación con un grupo de control (Tesis de doctorado inédito). Universidad de Castilla La Mancha.

Juárez, G. R. (2016). Situación de los niños, niñas y adolescentes privados de cuidados parentales en México. Entretextos, $22,1-14$.

Leloux-Opmeer, H., Kuiper, C., Swaab, H., \& Scholte, E. (2016). Characteristics of children in foster care, family-style group care, and residential care: a scoping review. The Journal of Child and Family Studies, 25, 2357-2371.

Leloux-Opmeer, H., Kuiper, C. H., Swaab, H. T., \& Scholte, E. M. (2017). Children referred to foster care, family-style group care, and residential care: (How) do they differ? Children and Youth Services Review, 77, 1-9.

Li, D., Chng, G. S., \& Chu, C. M. (2017). Comparing long-term placement outcomes of residential and family foster care: a meta-analysis. Trauma, Violence, \& Abuse. 20(5), 653-664. http://dx.doi.org/1524838017726427

Llanos, A. (2013). Salud, bienestar y desarrollo de niños y niñas con medida de separación familiar en protección infantil (Tesis doctoral inédito). Universidad de Oviedo.

Maganto, C. (2010). El reto del profesional evaluador: divergencias teóricas y convergencias técnicas. In C. Ibáñez (Org.), Técnicas de autoinforme en evaluación psicológica: la entrevista clínica (pp.53-80): Bilbao: Universidad del País Vasco.

Monzó-Fenollar, M., \& Ballespí-Solá, S. (2013). Psicopatología en adolescentes adoptados (Tesis de maestría inédito). Universidad Autónoma de Barcelona.

Moreno, J. H., Hernández, D., \& Suárez, M. A. (2016). Análisis bibliométrico de los problemas de comportamiento externalizado en niños y adolescentes de la ciudad de Bogotá (2003-2013). Psicogente, 19(35), 63-76. http://dx.doi. org/10.17081/psico.19.35.1209

Muzi, S., \& Bizzi, F. (2018). Externalizing problems and delinquent behaviors in residential-care male adolescents: associations with peer attachment and alexithymia. 4th International Congress of Clinical and Health Psychology on Children and Adolescents, Palma.

Pérez, C. (2016). Diferencias en autoestima, autoeficacia y satisfacción vital en adolescentes institucionalizados y adolescentes en contextos sociales de riesgo (Tesis de doctorado inédito). Universidad Pontificia de Comillas.

Poch, F. V., Ballabriga, M. C. J., Sans, J. C., Hidalgo, G. E., Solà, S. B., \& Doménech-Llaberia, E. (2008). Evaluación de la psicopatología del preescolar mediante el Early Childhood Inventory-4 (ECl-4): concordancia entre padres y maestros. Psicothema, 20(3), 481-486.

Rescorla, L., Ivanova, M. Y., Achenbach, T. M., Begocac, I. M. D., Chahed, M., Drugli, M. B., ... Zhang, E.Y. (2012). International epidemiology of child and adolescent psychopathology II: integration and applications of dimensional findings from 44 societies. Journal of the American Academy of Child \& Adolescent Psychiatry, 51, 1273-1283. http:// dx.doi.org/10.1016/j.jaac.2012.09.012

Rescorla, L. A., Althoff, R. R., Ivanova, M. Y., \& Achenbach, T. M. (2019). Effects of society and culture on parents' ratings of children's mental health problems in 45 societies. European Child \& Adolescent Psychiatry, 28, 1107-1115.

Sainero, A., Del Valle, J. F., López, M., \& Bravo, A. (2013). Exploring the specific needs of an understudied group: children with intellectual disability in residential child care. Children and Youth Services Review, 35(9), 1393-1399.

Sainero, A., Del Valle, J. F., \& Bravo, A. (2015). Detección de problemas de salud mental en un grupo especialmente vulnerable: niños y adolescentes en acogimiento residencial. Anales de Psicología, 31(2), 472-480.

Sánchez, M. (2015). Función ejecutiva en niños y niñas adoptados internacionalmente y su relación con el desarrollo socioemocional (Tesis de doctorado inédito). Universidad de Sevilla.

Steinberg, L., \& Morris, A. S. (2001). Adolescent development. Annual Review of Psychology, 52, 83-110.

Swinton, J. (2011). Adoptees and behavior problems: A meta-analysis (Tesis de doctorado inédito). Kansas State University.

Ulloa, N., Cova, F., \& Bustos, C. (2017). Nivel Socioeconómico y conductas externalizadas en preescolares: el rol del mediador parental. Revista Chilena de Pediatría, 88(3), 340-347.

Recibido: Abril 8, 2019

Versión final: Septiembre 23, 2019

Aprobado: Octubre 10, 2019 